\title{
Asteroseismology of hot pre-white dwarf stars: the case of the DOV stars PG 2131+066 and PG 1707+427, and the PNNV star NGC 1501
}

\author{
A. H. Córsico ${ }^{1,2,3}$, L. G. Althaus ${ }^{1,2,3}$, M. M. Miller Bertolami ${ }^{1,2,4}$, and E. García-Berro ${ }^{5,6}$ \\ 1 Facultad de Ciencias Astronómicas y Geofísicas, Universidad Nacional de La Plata, Paseo del Bosque S/N, (1900) La Plata, \\ Argentina \\ e-mail: [acorsico;althaus;mmiller]@fcaglp.unlp.edu.ar \\ 2 Instituto de Astrofísica La Plata, IALP, CONICET-UNLP, Argentina \\ 3 Member of the Carrera del Investigador Científico y Tecnológico, CONICET, Argentina \\ 4 Fellow of CONICET, Argentina \\ 5 Departament de Física Aplicada, Escola Politècnica Superior de Castelldefels, Universitat Politècnica de Catalunya, \\ Av. del Canal Olímpic, s/n, 08860 Castelldefels, Spain \\ e-mail: garcia@fa.upc.edu \\ ${ }^{6}$ Institute for Space Studies of Catalonia, c/Gran Capità 2-4, Edif. Nexus 104, 08034 Barcelona, Spain
}

Received 31 July 2008 / Accepted 26 March 2009

\begin{abstract}
Aims. We present an asteroseismological study on the two high-gravity pulsating PG 1159 (GW Vir or DOV) stars, PG $2131+066$ and PG $1707+427$, and on the pulsating [WCE] star NGC 1501. All of these stars have been intensively scrutinized through multi-site observations, so they have well resolved pulsation spectra.

Methods. We compute adiabatic $g$-mode pulsation periods on PG 1159 evolutionary models with stellar masses ranging from 0.530 to $0.741 M_{\odot}$. These models take into account the complete evolution of progenitor stars, through the thermally pulsing AGB phase, and born-again episode. We constrain the stellar mass of PG 2131+066, PG 1707+427, and NGC 1501 by comparing the observed period spacing with the asymptotic period spacing and with the average of the computed period spacings. We also employ the individual observed periods in search of representative seismological models for each star.

Results. We derive a stellar mass of $0.627 M_{\odot}$ for PG $2131+066,0.597 M_{\odot}$ for PG $1707+427$, and $0.571 M_{\odot}$ for NGC 1501 from a comparison between the observed period spacings and the computed asymptotic period spacings, and a stellar mass of $0.578 M_{\odot}$ for PG $2131+066,0.566 M_{\odot}$ for PG 1707+427, and $0.576 M_{\odot}$ for NGC 1501 by comparing the observed period spacings with the average of the computed period spacings. We also find, on the basis of a period-fit procedure, asteroseismological models representatives of PG $2131+066$ and PG 1707+427. These best-fit models are able to reproduce the observed period patterns of these stars with an average of the period differences of $\overline{\delta \Pi_{i}}=1.57 \mathrm{~s}$ and $\overline{\delta \Pi_{i}}=1.75 \mathrm{~s}$, respectively. The best-fit model for PG $2131+066$ has an effective temperature $T_{\text {eff }}=102100 \mathrm{~K}$, a stellar mass $M_{*}=0.589 M_{\odot}$, a surface gravity $\log g=7.63$, a stellar luminosity and radius of $\log \left(L_{*} / L_{\odot}\right)=1.57$ and $\log \left(R_{*} / R_{\odot}\right)=-1.71$, respectively, and a He-rich envelope thickness of $M_{\text {env }}=1.6 \times 10^{-2} M_{\odot}$. We derive a seismic distance $d \sim 830 \mathrm{pc}$ and a parallax $\pi \sim 1.2$ mas. The best-fit model for PG 1707+427, on the other hand, has $T_{\mathrm{eff}}=89500 \mathrm{~K}$, $M_{*}=0.542 M_{\odot}, \log g=7.53, \log \left(L_{*} / L_{\odot}\right)=1.40, \log \left(R_{*} / R_{\odot}\right)=-1.68$, and $M_{\text {env }}=2.5 \times 10^{-2} M_{\odot}$, and the seismic distance and parallax are $d \sim 730 \mathrm{pc}$ and $\pi \sim 1.4$ mas. Finally, we have been unable to find an unambiguous best-fit model for NGC 1501 on the basis of a period-fit procedure.

Conclusions. This work closes our short series of asteroseismological studies on pulsating pre-white dwarf stars. Our results demonstrate the usefulness of asteroseismology for probing the internal structure and evolutionary status of pre-white dwarf stars. In particular, asteroseismology is able to determine stellar masses of PG 1159 stars with an accuracy comparable or even better than spectroscopy.
\end{abstract}

Key words. stars: evolution - stars: interiors - stars: oscillations - white dwarfs

\section{Introduction}

Pulsating PG 1159 stars (also called GW Vir or DOV stars) are very hot hydrogen-deficient post-Asymptotic Giant Branch (AGB) stars with surface layers rich in helium, carbon, and oxygen (Werner \& Herwig 2006) that exhibit multiperiodic luminosity variations with periods ranging from 5 to $50 \mathrm{~min}$, attributable to non-radial pulsation $g$-modes (see Winget \& Kepler 2008 and Fontaine \& Brassard 2008, for a recent reviews). PG 1159 stars are thought to be the evolutionary link between Wolf-Rayet type central stars of planetary nebulae and most of the hydrogendeficient white dwarfs (Althaus et al. 2005). It is generally accepted that these stars have their origin in a born-again episode induced by a post-AGB helium thermal pulse - see Iben et al. (1983), Herwig et al. (1999), Lawlor \& MacDonald (2003), and Althaus et al. (2005) for recent references.

Recently, considerable observational effort has been invested to study pulsating PG 1159 stars. Particularly noteworthy are the works of Vauclair et al. (2002) on RX J2117.1+3412, Fu et al. (2007) on PG 0122+200, and Costa et al. (2008) and Costa \& Kepler (2008) on PG 1159-035. These stars have been monitored through long-term observations carried out with the Whole Earth Telescope (Nather et al. 1990). On the theoretical front, recent important progress in the numerical modeling of PG 1159 stars (Althaus et al. 2005; Miller Bertolami \& Althaus 2006, 2007a) has paved the way for unprecedented 
asteroseismological inferences for the mentioned stars (Córsico \& Althaus 2006; Córsico et al. 2007a,b, 2008). The new generation of PG 1159 evolutionary models of Miller Bertolami \& Althaus (2006) is derived from the complete evolutionary history of progenitor stars with different stellar masses and an elaborate treatment of the mixing and extra-mixing processes during the core helium burning and born-again phases. The success of these models at explaining the spread in surface chemical composition observed in PG 1159 stars (Miller Bertolami \& Althaus 2006), the short born-again times of V4334 Sgr (Miller Bertolami \& Althaus 2007b), and the location of the GW Vir instability strip in the $\log T_{\text {eff }}-\log g$ plane (Córsico et al. 2006) renders reliability to the inferences drawn from individual pulsating PG 1159 stars.

Besides the mentioned three well-studied pulsating PG 1159 stars, there exist two other variable stars of this class that have been also intensively scrutinized through the multi-site observations of the WET: PG 2131+066 and PG 1707+427. In addition, there is a variable central star of planetary nebula (PNNV), NGC 1501, which has been the subject of a nearly continuous photometric coverage from a global observing campaign by Bond et al. (1996). We briefly summarize the properties of these stars below.

PG 2131+066 was discovered as a variable star by Bond et al. (1984) with periods of about 414 and $386 \mathrm{~s}$, along with some other periodicities. On the basis of an augmented set of periods from WET data, Kawaler et al. (1995) obtained a precise mass determination of $M_{*}=0.61 M_{\odot}$, a luminosity of $10 L_{\odot}$, and a seismological distance from the Earth of $d=470 \mathrm{pc}$. Spectroscopic constraints of Dreizler \& Heber (1998), on the other hand, gave $M_{*}=0.55 M_{\odot}, T_{\text {eff }}=95000 \mathrm{~K}, 39.8 L_{\odot}$, and $\log g=7.5$ for PG $2131+066$. By using this updated determination of the effective temperature, Reed et al. (2000) refined the procedure of Kawaler et al. (1995) and found $M_{*}=0.608 M_{\odot}$, $L_{*}=26 L_{\odot}$ and $d=668 \mathrm{pc}$.

PG $1707+427$ was discovered to be a pulsator by Bond et al. (1984). Dreizler \& Heber (1998) obtained $T_{\text {eff }}=85000 \mathrm{~K}$, $\log g=7.5$, and then $M_{*}=0.54 M_{\odot}$ and $L_{*}=25 L_{\odot}$ were inferred from their spectroscopic study. Recently, Kawaler et al. (2004) reported the analysis of multi-site observations of PG 1707+427 obtained with WET. Preliminary seismic analysis by using 7 independent $\ell=1$ modes with periods between 334 and $910 \mathrm{~s}$ suggest an asteroseismological mass and luminosity of $0.57 M_{\odot}$ and $23 L_{\odot}$, respectively.

NGC 1501 was classified as a [WCE] star, an early low-mass Wolf Rayet-type PNNV with spectra dominated by strong helium and carbon emission lines (Werner \& Herwig 2006). The effective temperature and gravity of this star are $T_{\text {eff }}=134000 \mathrm{~K}$ and $\log g=6.0$ (Werner \& Herwig 2006). The variable nature of NGC 1501 was discovered by Bond \& Ciardullo (1993). The star shows ten periodicities ranging from $5200 \mathrm{~s}$ down to $1154 \mathrm{~s}$, although the largest amplitude pulsations occur between $1154 \mathrm{~s}$ and $2000 \mathrm{~s}$. Based on period-spacing data, Bond et al. (1996) found a stellar mass of $0.53 \pm 0.03 M_{\odot}$ for NGC 1501 .

In this work we complete our small survey of asteroseismological inferences on pulsating PG 1159 stars - see Córsico et al. (2007a,b, 2008) for the previous studies of this series - by performing a detailed study of the GW Vir stars PG 2131+066 and PG 1707+427, and the [WCE] star NGC 1501. We employ the same stellar models and numerical tools as in our previous works. In particular, we go beyond the mere use of the observed period-spacing data by performing, in addition, detailed period-to-period fits on the pulsation spectrum of these stars. In our approach, we take full advantage of the state-of-the-art
PG 1159 evolutionary models developed by Miller Bertolami \& Althaus (2006). The paper is organized as follows. In Sect. 2 we briefly describe our PG 1159 evolutionary models. In Sect. 3 we derive the stellar mass of PG 2131+066, PG 1707+427, and NGC 1501 by using the observed period-spacing data alone. In Sect. 4 we infer structural parameters of these stars by employing the individual observed periods. In this section we derive asteroseismological models representative of PG 2131+066 and PG 1707+427 (4.1), and discuss their main structural and pulsational characteristics (4.2). In Sect. 5 we compare the results of the present paper with those of the asteroseismological study of Córsico \& Althaus (2006, hereinafter CA06). Finally, in Sect. 6 we summarize our main results and make some concluding remarks.

\section{Evolutionary models and numerical tools}

The pulsation analysis presented in this work relies on a new generation of stellar models that take into account the complete evolution of PG 1159 progenitor stars. Specifically, the stellar models were extracted from the evolutionary calculations recently presented by Althaus et al. (2005), Miller Bertolami \& Althaus (2006), and Córsico et al. (2006), who computed the complete evolution of model star sequences with initial masses on the ZAMS ranging from 1 to $3.75 M_{\odot}$. All of the post-AGB evolutionary sequences were computed using the LPCODE evolutionary code (Althaus et al. 2005) and were followed through the very late thermal pulse (VLTP) and the resulting born-again episode that gives rise to the $\mathrm{H}$-deficient, $\mathrm{He}-, \mathrm{C}-$, and $\mathrm{O}$-rich composition characteristic of PG 1159 stars. The masses of the resulting remnants are $0.530,0.542,0.556,0.565,0.589,0.609$, 0.664 , and $0.741 M_{\odot}$. For details about the input physics and evolutionary code used, and the numerical simulations performed to obtain the PG 1159 evolutionary sequences employed here, we refer the interested reader to the works by Althaus et al. (2005) and Miller Bertolami \& Althaus (2006, 2007a).

We computed $\ell=1 g$-mode adiabatic pulsation periods with the same numerical code and methods we employed in our previous works, see Córsico \& Althaus (2006) for details. In addition, we performed nonadiabatic computations with the help of the code employed in Córsico et al. (2006) to evaluate the pulsational stability of the asteroseismological models presented in Sect. 4. We analyzed about 3000 PG 1159 models covering a wide range of effective temperatures $\left(5.4 \gtrsim \log \left(T_{\text {eff }}\right) \gtrsim 4.8\right)$ and luminosities $\left(0 \lesssim \log \left(L_{*} / L_{\odot}\right) \lesssim 4.2\right)$, and a range of stellar masses $\left(0.530 \leq M_{*} / M_{\odot} \leq 0.741\right)$.

\section{Mass determination from the observed period spacing}

In this section we constrain the stellar mass of PG 2131+066, PG $1707+427$, and NGC 1501 by comparing the asymptotic period spacing and the average of the computed period spacings with the observed period spacing. These approaches take full advantage of the fact that the period spacing of PG 1159 pulsators depends primarily on the stellar mass, and the dependence on the luminosity and the He-rich envelope mass fraction is negligible (Kawaler \& Bradley 1994; Córsico \& Althaus 2006). Most of the published asteroseismological studies on PG 1159 stars rely on the asymptotic period spacing to infer the total mass of GW Vir pulsators, the notable exception being the works by Reed et al. (2000) for PG 2131+066, Córsico et al. (2007a) for RX J2117.1+3412, Córsico et al. (2007b) for PG 0122+200, 
Table 1. Stellar masses for all of the intensively studied pulsating PG 1159 stars, including also one pulsating [WCE] star. All masses are in solar units.

\begin{tabular}{lcccclc}
\hline \hline Star & $\Delta \Pi_{\ell}^{\mathrm{a}}$ & $\overline{\Delta \Pi_{\ell}}$ & $\begin{array}{c}\text { Approximate } \\
\text { formula }\end{array}$ & Period fit & $\begin{array}{l}\text { Pulsations } \\
\text { (other works) }\end{array}$ & Spectroscopy \\
\hline NGC 1501 & $0.571^{a}$ & $0.576^{a}$ & $0.530^{a}$ & $\overline{-}$ & $0.55^{j}$ (asymptotic analysis) & 0.56 \\
RX J2117.1+3412 & $0.568^{b}$ & $0.560^{b}$ & $0.525^{a}$ & $0.565^{b}$ & $0.56^{h}$ (asymptotic analysis) & 0.72 \\
PG 1159-035 & $0.577-0.585^{d}$ & $0.561^{d}$ & $0.570^{a}$ & $0.565^{d}$ & $0.59^{i}$ (asymptotic analysis) & 0.54 \\
PG 2131+066 & $0.627^{a}$ & $0.578^{a}$ & $0.609^{a}$ & $0.589^{a}$ & $0.61^{e}$ (period fit) & 0.55 \\
PG 1707+427 & $0.597^{a}$ & $0.566^{a}$ & $0.587^{a}$ & $0.542^{a}$ & $0.57^{g}$ (asymptotic analysis) & 0.53 \\
PG 0122+200 & $0.625^{c}$ & $0.567^{c}$ & $0.593^{a}$ & $0.566^{c}$ & $0.69^{f}$ (asymptotic analysis) & 0.53 \\
\hline
\end{tabular}

Notes: ${ }^{a}$ This work. ${ }^{b}$ Córsico et al. (2007a). ${ }^{c}$ Córsico et al. (2007b). ${ }^{d}$ Córsico et al. (2008). ${ }^{e}$ Reed et al. (2000). ${ }^{f}$ Fu et al. (2007). ${ }^{g}$ Kawaler et al. (2004). ${ }^{h}$ Vauclair et al. (2002). ${ }^{i}$ Costa et al. (2008). ${ }^{j}$ Bond et al. (1996).

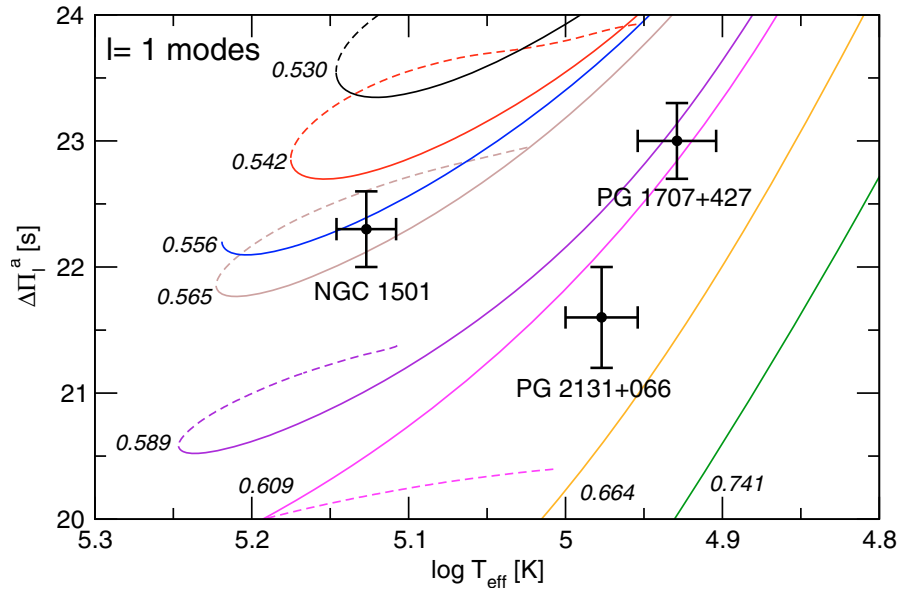

Fig. 1. The dipole asymptotic period spacing in terms of the effective temperature. Numbers along each curve denote the stellar mass (in solar units). Dashed (solid) lines correspond to evolutionary stages before (after) the turning point at the maxima effective temperature of each track. Also shown are the locations of PG $2131+066$, PG 1707+427, and NGC 1501.

and Kawaler \& Bradley (1994) and Córsico et al. (2008) for PG 1159-035. To assess the total mass of NGC 1501 we have considered the high-luminosity regime of the evolutionary sequences, while for PG $2131+066$ and PG $1707+427$ we have focused on the stages following the "evolutionary knee" for the PG 1159 stars, i.e. the low-luminosity regime.

\subsection{First method: comparing the observed period spacing $\left(\Delta \Pi_{\ell}^{\mathrm{O}}\right)$ with the asymptotic period spacing $\left(\Delta \Pi_{\ell}^{\mathrm{a}}\right)$}

Figure 1 displays the asymptotic period spacing for $\ell=1$ modes as a function of the effective temperature for different stellar masses. Also shown in this diagram is the location of PG 2131+066, with $T_{\text {eff }}=95 \pm 5 \mathrm{kK}$ (Dreizler \& Heber 1998), and $\Delta \Pi_{\ell=1}^{\mathrm{O}}=21.6 \pm 0.4 \mathrm{~s}$ (Reed et al. 2000), PG 1707+427, with $T_{\mathrm{eff}}=85 \pm 5 \mathrm{kK}$ (Dreizler \& Heber 1998), and $\Delta \Pi_{\ell=1}^{\mathrm{O}}=$ $23.0 \pm 0.3 \mathrm{~s}$ (Kawaler et al. 2004), and NGC 1501, with $T_{\text {eff }}=$ $134 \pm 5 \mathrm{kK}$ (Werner \& Herwig 2006), and $\Delta \Pi_{\ell=1}^{\mathrm{O}}=22.3 \pm 0.3 \mathrm{~s}$ (Bond et al. 1996). The asymptotic period spacing is computed as $\Delta \Pi_{\ell}^{\mathrm{a}}=\Pi_{0} / \sqrt{\ell(\ell+1)}$, where

$\Pi_{0}=2 \pi^{2}\left[\int_{r_{1}}^{r_{2}}(N / r) \mathrm{d} r\right]^{-1}$

and $N$ is the Brunt-Väisälä frequency (Tassoul et al. 1990). From the comparison between the observed $\Delta \Pi_{\ell=1}^{\mathrm{O}}$ and $\Delta \Pi_{\ell=1}^{\mathrm{a}}$ we found a stellar mass of $0.627 M_{\odot}$ for PG 2131+066, $0.597 M_{\odot}$ for PG $1707+427$, and $0.571 M_{\odot}$ for NGC 1501 (second column in Table 1).

The method employed here is computationally inexpensive and widely used because it does not involve pulsational calculations. However, we must emphasize that the derivation of the stellar mass using the asymptotic period spacing may not be entirely reliable in pulsating PG 1159 stars that pulsate with modes characterized by low and intermediate radial orders (see Althaus et al. 2007). This is particularly true for PG 2131+066 and PG $1707+427$. This shortcoming of the method is due to that the asymptotic predictions are strictly valid in the limit of very high radial order (long periods) and for chemically homogeneous stellar models, while PG 1159 stars are supposed to be chemically stratified and characterized by strong chemical gradients built up during the progenitor star life. A more realistic approach to infer the stellar mass of PG 1159 stars is presented below.

\subsection{Second method: comparing the observed period spacing $\left(\Delta \Pi_{\ell}^{\mathrm{O}}\right)$ with the average of the computed period spacings $\overline{\left(\Delta \Pi_{\ell}\right)}$}

The average of the computed period spacings is assessed as $\overline{\Delta \Pi_{\ell}}=(N-1)^{-1} \sum_{k} \Delta \Pi_{k}$, where the "forward" period spacing is defined as $\Delta \Pi_{k}=\Pi_{k+1}-\Pi_{k}$ ( $k$ being the radial order) and $N$ is the number of computed periods laying in the range of the observed periods. For PG $2131+066, \Pi_{k} \in[340,600] \mathrm{s}$, according to Kawaler et al. (1995); for PG 1707+427, $\Pi_{k} \in[330,920] \mathrm{s}$, according to Kawaler et al. (2004); and for NGC 1501, $\Pi_{k} \in$ [1150, 2000] s, according to Bond et al. (1996).

This method is more reliable for the estimation of the stellar mass of PG 1159 stars than that described above because, provided that the average of the computed period spacings is evaluated at the appropriate range of periods, the approach is appropriate for the regimes of short, intermediate and long periods (i.e., $\forall k$ ) as well. When the average of the computed period spacings is taken over a range of periods characterized by high $k$ values, then the predictions of the present method become closer to those of the asymptotic period spacing approach. On the other hand, the present method requires of detailed period computations, at variance with the method described in the above section. In addition, we note that both methods for assessing the stellar mass rely on the spectroscopic effective temperature, and the results are unavoidably affected by its associated uncertainty.

In Fig. 2 we show the run of average of the computed period spacings $(\ell=1)$ for PG $2131+066$, PG 1707+427, and NGC 1501 in terms of the effective temperature for all of our 


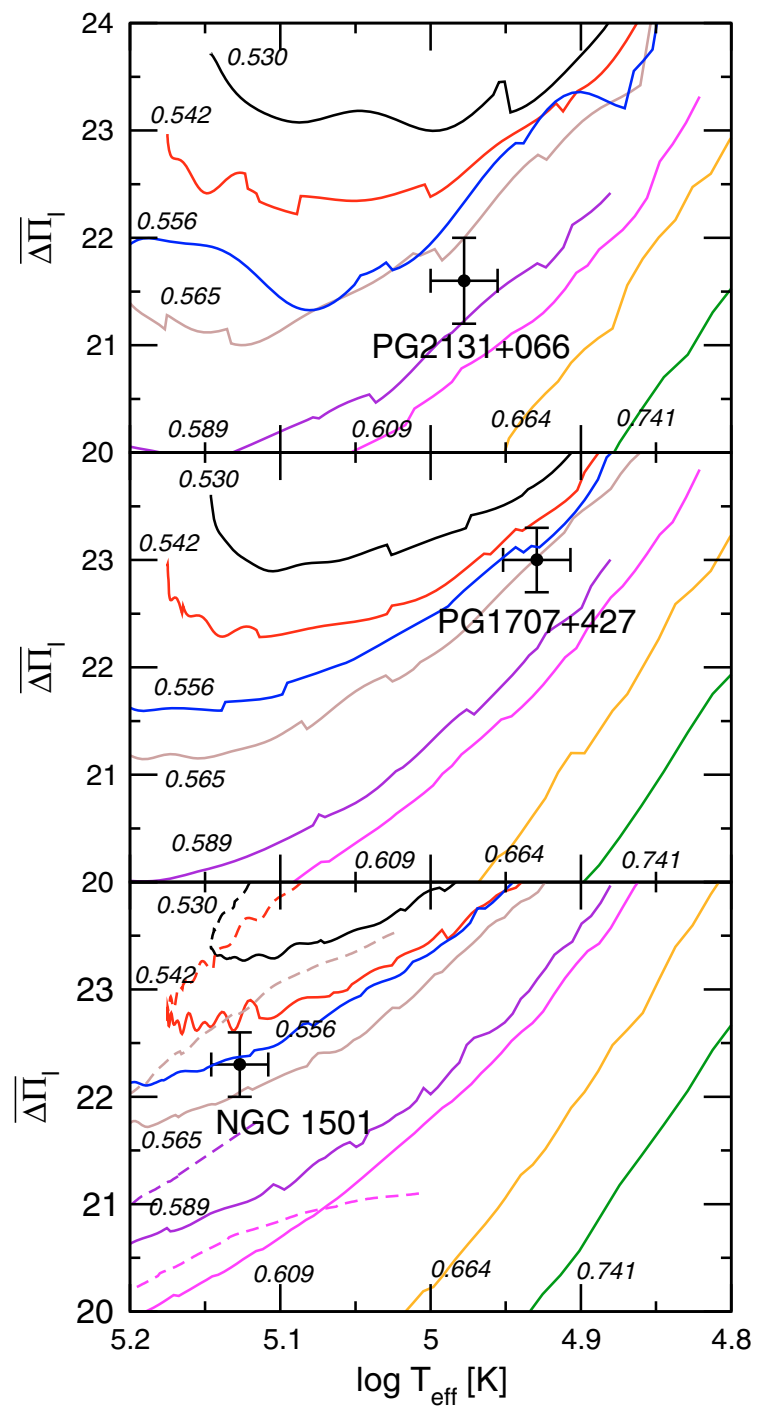

Fig. 2. Same as Fig. 1, but for the average of the computed period spacings. For PG $2131+066$ and PG $1707+427$ only the stages after the "evolutionary knee" have been plotted. As in Fig. 1, we infer the mass of NGC 1501 by considering the stages before the evolutionary knee (dashed lines).

PG 1159 evolutionary sequences. The run of $\overline{\Delta \Pi_{\ell}}$ depends on the range of periods on which the average of the computed period spacing is done. Note that the lines shown in Fig. 2 are very jagged and jumped. This is because that, for a given star, the average of the computed period spacings is evaluated for a fixed period interval, and not for a fixed $k$-interval ${ }^{1}$.

By adopting the effective temperature of PG 2131+066, PG $1707+427$, and NGC 1501 as given by spectroscopy we found a stellar mass of $0.578 M_{\odot}, 0.566 M_{\odot}$, and $0.576 M_{\odot}$, respectively. Our results are shown in the third column of Table 1. These values are $8.5 \%$ (for PG 2131+066) and $5.5 \%$ (for PG 1707+427) smaller than those derived through

\footnotetext{
${ }^{1}$ As the star evolves towards higher (lower) effective temperatures, the periods generally decrease (increase) with time. At a given $T_{\text {eff }}$, there are $N$ computed periods laying in the chosen period interval. Later when the model has evolved enough (heated or cooled) it is possible that the accumulated period drift nearly matches the period separation between adjacent modes $(|\Delta k|=1)$. In these circumstances, the number of periods laying in the chosen (fixed) period interval is $N \pm 1$, and $\overline{\Delta \Pi_{\ell}}$ exhibits a little jump.
}

the asymptotic period spacing, showing once again that the asymptotic approach overestimates the stellar mass of PG 1159 stars that, like PG $2131+066$ and PG 1707+427, exhibit short and intermediate pulsation periods (see Althaus et al. 2008a). On the contrary, there is a very small discrepancy (in the opposite direction) of $\sim 0.9 \%$ for the case of NGC 1501, showing that in the long-period regime the results for the stellar mass obtained using the asymptotic period spacing and the average of the computed period spacings nicely agree each other. A similar situation is found in the case of RX J2117.1+3412 (Córsico et al. 2007a).

\subsection{Third method: using an approximate formula}

To compare with previous works, we make an additional estimation of the stellar mass of PG $2131+066$, PG 1707+427, and NGC 1501 using the approximate expression for the overall structure parameter $\Pi_{0}$ derived by Kawaler \& Bradley (1994):

$\Pi_{0} \approx 15.5\left(\frac{M_{*}}{M_{\odot}}\right)^{-1 / 3}\left(\frac{L_{*}}{100 L_{\odot}}\right)^{-0.035}\left(\frac{q_{y}}{10^{-3}}\right)^{-0.00012}$

where $q_{y}$ is the He-rich envelope mass fraction. This expression is derived by considering the dependence of the asymptotic period spacing on the total mass, stellar luminosity, and thickness of the He-rich outer envelope for a large grid of "quasi evolutionary" PG 1159 models in the luminosity range $1.6 \lesssim$ $\log \left(L_{*} / L_{\odot}\right) \lesssim 3.0$. Both the present method and the method described in Sect. 3.1 are almost equivalent because they are based on the asymptotic period spacing.

Due to the very weak dependence of $\Pi_{0}$ on $q_{y}$, we arbitrarily fix it to a value of $10^{-2}$. Since the luminosity is not known at the outset, we can compute it as $L_{*}=4 \pi \sigma R_{*}^{2} T_{\text {eff }}^{4}$, where $R_{*}^{2}=G M_{*} / g$. We use the values of $g$ and $T_{\text {eff inferred through }}$ spectroscopy. Assuming that $\Pi_{0}$ is known from the observed period spacing $\left(\Pi_{0} \sim \sqrt{\ell(\ell+1)} \Delta \Pi_{\ell}^{\mathrm{O}}\right)$, we obtain an estimation of the stellar mass from Eq. (2). Our results are shown in the fourth column of Table 1. For PG 2131+066 and PG 1707+427 the stellar masses obtained in this way are in very good agreement with our values derived from the asymptotic period spacing (first row in Table 1). This is not an unexpected result because, as mentioned, the expression of Kawaler \& Bradley (1994) is also based on the asymptotic period spacing. The slight differences found (below $\approx 2.5 \%$ ) could be due to differences in the modeling of PG 1159 stars. For NGC 1501, instead, there is a substantial difference $(\approx 7 \%)$ between the prediction of this formula and our value inferred from the asymptotic period spacing. This could be due to the inadequacy of the formula of Kawaler \& Bradley (1994) for the high-luminosity regime characterizing the evolutionary status of NGC 1501.

\section{Constraints from the individual observed periods}

In this approach we seek pulsation models that best match the individual pulsation periods of PG $2131+066$, PG 1707+427, and NGC 1501. For the three stars, we assume that all of the observed periods correspond to $\ell=1$ modes because the observed period spacings and the frequency splittings by rotation are consistent with $\ell=1$ (Kawaler et al. 1995, 2004; Bond et al. 1996). To measure the goodness of the match between the theoretical pulsation periods $\left(\Pi_{k}^{\mathrm{T}}\right)$ and the observed individual periods $\left(\Pi_{i}^{\mathrm{O}}\right)$, we follow the same $\chi^{2}$ procedure as in our previous 
Table 2. Observed and theoretical $(\ell=1)$ periods of the best-fit model for PG $2131+066\left(M_{*}=0.589 M_{\odot}, T_{\text {eff }}=102179 \mathrm{~K}, \log \left(L_{*} / L_{\odot}\right)=\right.$ 1.57). Periods are in seconds and rates of period change (theoretical) are in units of $10^{-12} \mathrm{~s} / \mathrm{s}$. The observed periods are taken from Kawaler et al. (1995).

\begin{tabular}{ccccc}
\hline \hline$\Pi_{i}^{\mathrm{O}}$ & $\Pi_{k}^{\mathrm{T}}$ & $k$ & $\dot{\Pi}_{k}^{\mathrm{T}}$ & Unstable \\
\hline 341.45 & 341.88 & 14 & 2.04 & yes \\
- & 363.66 & 15 & 3.90 & yes \\
384.27 & 383.94 & 16 & 2.60 & yes \\
403.93 & 403.30 & 17 & 3.30 & yes \\
426.36 & 426.48 & 18 & 4.14 & yes \\
450.28 & 445.31 & 19 & 2.89 & yes \\
462.39 & 465.91 & 20 & 4.53 & yes \\
- & 488.81 & 21 & 4.11 & no \\
507.91 & 507.91 & 22 & 3.79 & no \\
\hline
\end{tabular}

works - see, e.g, Córsico et al. (2007a) and Townsley et al. (2004). Specifically, we employ the quality function defined as

$\chi^{2}\left(M_{*}, T_{\text {eff }}\right)=\frac{1}{n} \sum_{i=1}^{n} \min \left[\left(\Pi_{i}^{\mathrm{O}}-\Pi_{k}^{\mathrm{T}}\right)^{2}\right]$

where $n$ is the number of observed periods. The observed periods are shown in the first column of Tables 2 and 3 for PG 2131+066 and PG $1707+427$, and in Table 7 of Bond et al. (1996) for NGC 1501.

Next, we briefly explain the procedure we follow to found a model representative of a target star. For a given model (characterized by a given $T_{\text {eff }}$ ) corresponding to an evolutionary sequence of stellar mass $M_{*}$, we consider the first observed period of the list, namely $\Pi_{1}^{\mathrm{O}}$, and compute the successive squared differences $\left(\Pi_{1}^{\mathrm{O}}-\Pi_{k}^{\mathrm{T}}\right)^{2}$, where the radial order $k$ varies from 1 to a given maximum value, $k_{\max }$, which corresponds to a theoretical period far longer that the maximum period observed in the star. Next, we retain the minor squared difference, and then we repeat the procedure but this time considering the second observed period, namely $\Pi_{2}^{\mathrm{O}}$. After the minor squared difference associated with this observed period is stored, we proceed with the next observed period, and so until the minor squared difference associated with the last observed period $\left(\Pi_{n}^{\mathrm{O}}\right)$ is stored. The next step is to calculate the sum of these differences and then obtain the value of $\chi^{2}\left(M_{*}, T_{\text {eff }}\right)$ for the model under consideration. It is worth mentioning that with this algorithm, the value of $\chi^{2}\left(M_{*}, T_{\text {eff }}\right)$ does not depend on the particular order in which the observed periods are fitted. The complete algorithm is repeated for all of the models of the sequence, and then, a curve of $\chi^{2}\left(M_{*}, T_{\text {eff }}\right)$ versus $T_{\text {eff }}$ is obtained for the complete sequence. This procedure is carried out for all of our sequences. For each star of interest, the PG 1159 model that shows the lowest value of $\chi^{2}$ is adopted as the "best-fit model".

\subsection{The search for the best-fit models}

We evaluate the function $\chi^{2}=\chi^{2}\left(M_{*}, T_{\text {eff }}\right)$ for stellar masses of $0.530,0.542,0.556,0.565,0.589,0.609,0.664$, and $0.741 M_{\odot}$. For the effective temperature we employed a much finer grid $\left(\Delta T_{\text {eff }}=10-30 \mathrm{~K}\right)$. The quantity $\left(\chi^{2}\right)^{-1}$ in terms of the effective temperature for different stellar masses is shown in Fig. 3 for PG 2131+066 (upper panel), PG 1707+427 (middle panel), and NGC 1501 (lower panel), together with the corresponding spectroscopic effective temperatures. We prefer to show in our plots the quantity $\left(\chi^{2}\right)^{-1}$ instead $\chi^{2}$ in order to emphasize
Table 3. Same as Table 2, but for the best-fit model for PG 1707+427 $\left(M_{*}=0.542 M_{\odot}, T_{\text {eff }}=89504 \mathrm{~K}, \log \left(L_{*} / L_{\odot}\right)=1.40\right)$. The observed periods are taken from Kawaler et al. (2004).

\begin{tabular}{ccccc}
\hline \hline$\Pi_{i}^{\mathrm{O}}$ & $\Pi_{k}^{\mathrm{T}}$ & $k$ & $\dot{\Pi}_{k}^{\mathrm{T}}$ & Unstable \\
\hline 334.62 & 333.26 & 12 & 2.13 & yes \\
- & 355.73 & 13 & 1.19 & yes \\
- & 378.53 & 14 & 2.17 & yes \\
- & 401.64 & 15 & 1.51 & yes \\
- & 423.68 & 16 & 1.84 & yes \\
448.07 & 447.31 & 17 & 2.46 & yes \\
- & 469.26 & 18 & 1.49 & yes \\
494.39 & 492.56 & 19 & 2.76 & yes \\
- & 516.50 & 20 & 2.61 & yes \\
536.41 & 537.67 & 21 & 1.66 & yes \\
- & 562.32 & 22 & 3.30 & yes \\
- & 584.78 & 23 & 2.37 & yes \\
- & 606.62 & 24 & 2.73 & yes \\
- & 632.11 & 25 & 3.25 & yes \\
- & 653.15 & 26 & 2.99 & yes \\
677.89 & 677.38 & 27 & 3.43 & yes \\
- & 701.20 & 28 & 3.03 & yes \\
726.02 & 722.82 & 29 & 3.95 & no \\
745.78 & 747.58 & 30 & 3.42 & no \\
- & 770.93 & 31 & 3.71 & no \\
- & 793.13 & 32 & 4.45 & no \\
- & 817.86 & 33 & 3.61 & no \\
- & 841.70 & 34 & 4.64 & no \\
- & 863.34 & 35 & 3.91 & no \\
- & 888.59 & 36 & 5.03 & no \\
909.05 & 912.34 & 37 & 4.21 & no \\
\hline
\end{tabular}

the location of models providing good agreements between observed and theoretical periods. As mentioned, the goodness of the match between the observed and theoretical periods is reflected by the value of $\chi^{2}$. The lower the value of $\chi^{2}$, the better the period match. We will consider - admittedly somewhat arbitrarily - that a peak in the quality function with $\chi^{2} \lesssim 5$ (that is, $\left.\left(\chi^{2}\right)^{-1} \gtrsim 0.2\right)$ is a good match between the theoretical and the observed periods.

For PG $2131+066$ we find one strong maximum of $\left(\chi^{2}\right)^{-1}$ for a model with $M_{*}=0.589 M_{\odot}$ and $T_{\text {eff }} \approx 102 \mathrm{kK}$. Such a pronounced maximum in the inverse of $\chi^{2}$ implies an excellent agreement between the theoretical and observed periods. Another much less pronounced maxima, albeit at effective temperatures closer to the spectroscopic estimation for PG 2131+066, are encountered for $M_{*}=0.609 M_{\odot}$ at $T_{\text {eff }} \approx$ $92.4 \mathrm{kK}$ and $M_{*}=0.565 M_{\odot}$ at $T_{\text {eff }} \approx 99.8 \mathrm{kK}$. However, because the agreement between observed and theoretical periods for these models are substantially poorer than for the one with $M_{*}=0.589 M_{\odot}$, we adopt this last model as the best-fit asteroseismological model. A detailed comparison of the observed $\ell=1, m=0$ periods in PG $2131+066$ with the theoretical periods of the best-fit model is provided in Table 2 . The high quality of our period fit is quantitatively reflected by the average of the absolute period differences

$\overline{\delta \Pi_{i}}=\frac{1}{n} \sum_{i=1}^{n}\left|\delta \Pi_{i}\right|$

where $\delta \Pi_{i}=\Pi_{i}^{\mathrm{O}}-\Pi_{k}^{\mathrm{T}}$ and by the root-mean-square residual

$\sigma_{\delta \Pi_{i}}=\sqrt{\frac{\left(\sum_{i=1}^{n}\left|\delta \Pi_{i}\right|^{2}\right)}{n}}$ 


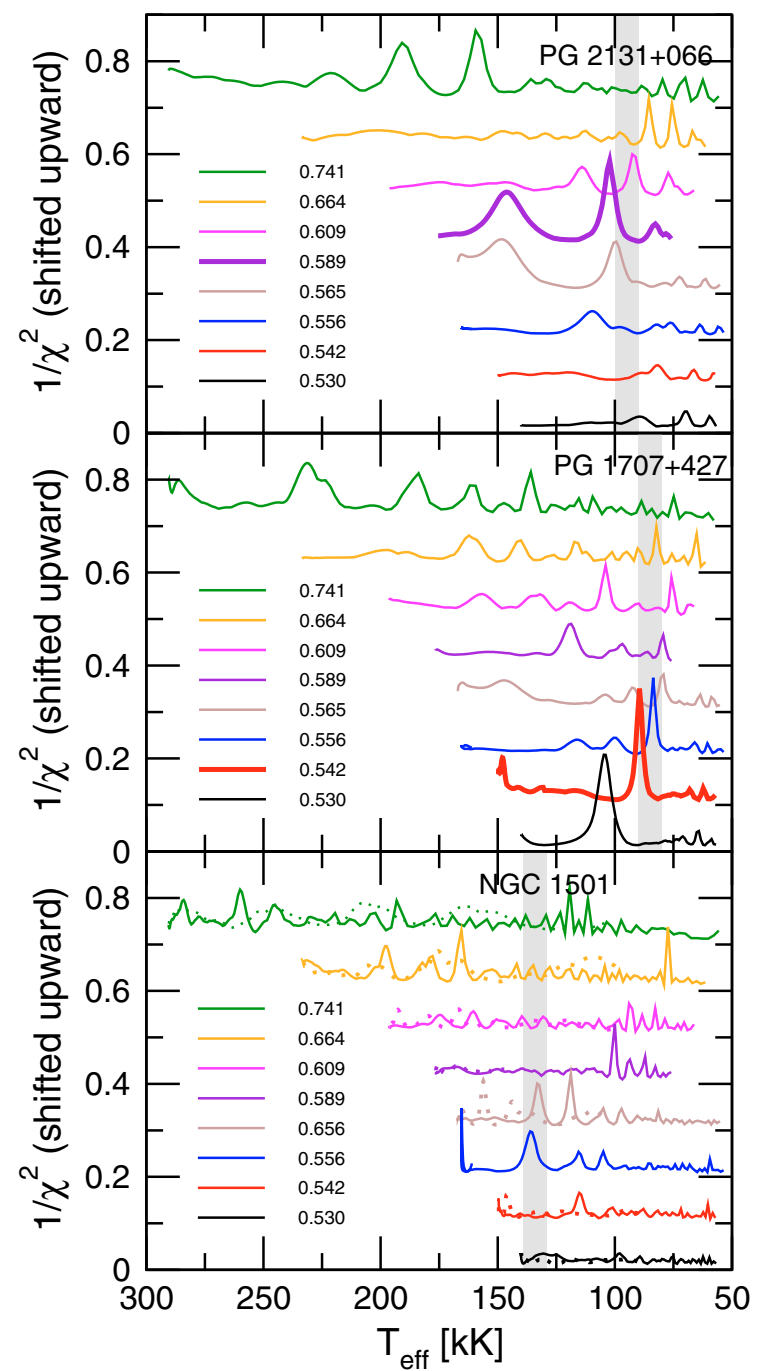

Fig. 3. The inverse of the quality function of the period fit in terms of the effective temperature (see text for details). The vertical grey strip indicates the spectroscopic $T_{\text {eff }}$ and its uncertainties. The curves have been arbitrarily shifted upward (with a step of 0.1). For NGC 1501, the stages before the evolutionary knee are displayed with dotted lines.

Note that $\sigma_{\delta \Pi_{i}}$ is simply the function $\sqrt{\chi^{2}}$ evaluated at the best-fit model. For the best-fit model of PG $2131+066$ we obtain $\overline{\delta \Pi_{i}}=$ $1.57 \mathrm{~s}$ and $\sigma_{\delta \Pi_{i}}=2.32 \mathrm{~s}$, which are indeed very small.

For PG $1707+427$ we find one strong maximum of $\left(\chi^{2}\right)^{-1}$ for a model with $M_{*}=0.542 M_{\odot}$ and $T_{\text {eff }} \approx 89.5 \mathrm{kK}$, an effective temperature compatible with the spectroscopic determination. Another somewhat less pronounced maximum is found for a model with $M_{*}=0.556 M_{\odot}$ and $T_{\text {eff }} \approx 83.6 \mathrm{kK}$. Despite the fact that this model has an effective temperature very close to the spectroscopic one, we choose the model with $M_{*}=0.542 M_{\odot}$ as the best-fit model for PG $1707+427$, because the period fit is characterized by a better quality. Table 3 shows a comparison between observed $\ell=1, m=0$ and computed periods of the bestfit model. We found in this case $\overline{\delta \Pi_{i}}=1.75 \mathrm{~s}$ and $\sigma_{\delta \Pi_{i}}=1.99 \mathrm{~s}$.

The situation for NGC 1501 is markedly different than for PG 2131+066 and PG 1707+427. Indeed, as clearly shown in the lower panel of Fig. 3, for this star the $\left(\chi^{2}\right)^{-1}$ function exhibits numerous local maxima at several values of the effective temperature and the stellar mass $\left(M_{*} / M_{\odot}=0.556,0.565\right.$, $0.589,0.664$, and 0.741$)$ that have roughly the same amplitudes, making virtually impossible to isolate a clear and unambiguous seismological solution. Thus, for NGC 1501 we are unable to find a best-fit seismological model. This could be, in part, due to the fact that the periods detected in NGC 1501 can be associated to eigenmodes with radial orders $k$ quite different from each other (with a mean spacing of $\overline{\Delta k} \gtrsim 6$ ), in such a way that it is easy to find numerous models (characterized by strongly different $T_{\text {eff }}$ and $M_{*}$ ) that reproduce to a some extent the observed period spectrum of NGC 1501 . It could also be that the impossibility to find a best fit model would be reflecting a different evolutionary history for NGC 1501 than that assumed in this work for our PG 1159 sequences.

The fourth column in Tables 2 and 3 shows the rates of period change associated with the fitted modes for PG 2131+066 and PG $1707+427$, respectively. Our calculations predict that all of the pulsation periods increase with time ( $\dot{\Pi}>0)$, in accordance with the decrease of the Brunt-Väisälä frequency in the core of the models induced by cooling. At the effective temperatures of PG 2131+066 and PG 1707+427, cooling has the largest effect on $\dot{\Pi}$, while gravitational contraction, which should result in a decrease of periods with time, becomes negligible and no longer affects the pulsation periods, except for the case of modes trapped in the envelope. Until now, the only secure measurements of $\dot{\Pi}$ in pre-white dwarf stars are those of PG 1159-035, the prototype of the class, by Costa et al. (1999) and more recently by Costa \& Kepler (2008). In this last paper the authors obtained a mix of positive and negative values of $\dot{\Pi}$, indicating that for that star gravitational contraction is still important. In principle, the needed time interval that the observational data should cover in order to reach a measurement of a Пं in PG 1159 stars like PG $2131+066$ and PG $1707+427$ is of about ten years. Unfortunately, no future observations in the short term that could allow a determination of $\dot{\Pi}$ for these stars and thus to check the predictions of our models are foreseen.

Finally, the last column in Tables 2 and 3 gives information about the pulsational stability/instability nature of the modes associated with the periods fitted to the observed ones. Full nonadiabatic calculations employing the pulsation code described in Córsico et al. (2006) indicate that, for the case of PG 2131+066, all the fitted modes except one (that with period $507.9 \mathrm{~s}$ ) are predicted to be unstable. For the case of PG $1707+427$, our nonadiabatic computations are able to explain the existence of periodicities in the range $330 \lesssim \Pi^{\mathrm{O}} \lesssim 680 \mathrm{~s}$ only, while they fail to predict pulsational instability for the observed modes with periods at 726,746 , and 909 s.

\subsection{Characteristics of the best-fit models for $P G 2131+066$ and $P G 1707+427$}

The main features of our best-fit model for PG 2131+066 are summarized in Table 4, where we also provide the parameters of the star extracted from other published studies. Specifically, the second column corresponds to spectroscopic results from Werner \& Herwig (2006), whereas the third and fourth columns present results from the pulsation studies of Kawaler et al. (1995) and Reed et al. (2000), and from the asteroseismological model of this work, respectively. The number in parenthesis is the spectroscopic estimation of the stellar mass employing the evolutionary tracks of Miller Bertolami \& Althaus (2006). In the present work, errors in $T_{\text {eff }}$ and $\log \left(L_{*} / L_{\odot}\right)$ are estimated from the width of the maximum in the function $\chi^{2}$ with respect $T_{\text {eff }}$ and $\log \left(L_{*} / L_{\odot}\right)$, respectively. The error in the stellar mass comes from the grid resolution in $M_{*}$. Errors in the rest 
Table 4. The main characteristics of PG 2131+066.

\begin{tabular}{lccc}
\hline \hline Quantity & Spectroscopy & Seismology & This work \\
\hline$T_{\text {eff }}[\mathrm{kK}]$ & $95 \pm 5$ & - & $102.18_{-2.8}^{+3.0}$ \\
$M_{*}\left[M_{\odot}\right]$ & $0.58 \pm 0.1$ & $0.608 \pm 0.01$ & $0.589_{-0.024}^{+0.020}$ \\
& $(0.55 \pm 0.1)$ & & \\
$\log g\left[\mathrm{~cm} / \mathrm{s}^{2}\right]$ & $7.5 \pm 0.5$ & - & $7.63_{-0.14}^{+0.12}$ \\
$\log \left(L_{*} / L_{\odot}\right)$ & - & $1.41 \pm 0.5$ & $1.57_{-0.06}^{+0.07}$ \\
$\log \left(R_{*} / R_{\odot}\right)$ & - & -1.73 & $-1.71_{-0.05}^{+0.06}$ \\
$M_{\text {env }}\left[M_{\odot}\right]$ & - & $3.8 \times 10^{-3}$ & 0.016 \\
$M_{\mathrm{V}}[\mathrm{mag}]$ & - & $7.69_{-0.18}^{+0.25}$ & $6.825_{-0.675}^{+0.655}$ \\
$M_{\text {bol }}[\mathrm{mag}]$ & - & - & $0.825_{-0.175}^{+0.155}$ \\
$A_{\mathrm{V}}[\mathrm{mag}]$ & - & - & 0.18 \\
$d[\mathrm{pc}]$ & - & $668_{-83}^{+78}$ & $830_{-224}^{+300}$ \\
$\pi[\mathrm{mas}]$ & - & $1.50 \pm 0.2$ & $1.2_{-0.3}^{+0.4}$ \\
\hline
\end{tabular}

of the quantities are derived from these values. The effective temperature of our best-fit model $\left(T_{\text {eff }}=102180 \mathrm{~K}\right)$ is somewhat higher than - but still compatible with - the spectroscopic value $\left(T_{\text {eff }}=95000 \pm 5000 \mathrm{~K}\right)$. On the other hand, the total mass of the best-fit model $\left(M_{*}=0.589 M_{\odot}\right)$ is in agreement with the value derived from the average of the computed period spacings $\left(M_{*} \sim 0.578 M_{\odot}\right)$, but at odds ( $6 \%$ smaller) with that inferred from the asymptotic period spacing $\left(M_{*}=0.627 M_{\odot}\right)$ (see Table 1). Also, the $M_{*}$ value of our best-fit model is substantially larger than the spectroscopic mass of $0.55 M_{\odot}$ derived by Miller Bertolami \& Althaus (2006), but very similar to $0.58 M_{\odot}$ according to Werner \& Herwig (2006). A discrepancy between the asteroseismological and the spectroscopic values of $M_{*}$ is generally encountered among PG 1159 pulsators - see Córsico et al. (2006, 2007a,b). Until now, the asteroseismological mass of PG $2131+066$ has been about $11 \% \operatorname{larger}\left(\Delta M_{*} \approx 0.06 M_{\odot}\right)$ than the spectroscopic mass if we consider the early estimation for the seismological mass quoted by Reed et al. (2000) and the derivation of Miller Bertolami \& Althaus (2006) for the spectroscopic mass ${ }^{2}$. In light of the best-fit model derived in this paper, this discrepancy is slightly reduced to about $7 \%$ $\left(\Delta M_{*} \approx 0.04 M_{\odot}\right)$. Finally, our best-fit model for PG $2131+066$ is somewhat more luminous and less compact than what is suggested by the results of Reed et al. (2000).

The main properties of our best-fit model for PG 1707+427 are shown in Table 5. The second column corresponds to spectroscopic results from Werner \& Herwig (2006), whereas the third and fourth columns present results from the pulsation study of Kawaler et al. (2004) and from the asteroseismological model of this work, respectively. As for the case of PG 2131+066, the effective temperature of our best-fit model for PG 1707+427 is slightly larger than the spectroscopic measurement, but even in good agreement with it. Regarding the stellar mass, our bestfit model has $M_{*}=0.542 M_{\odot}$, which is in agreement with the value derived from the average of the computed period spacings $\left(M_{*} \sim 0.566 M_{\odot}\right)$, but at odds $(\sim 9 \%$ lower $)$ with that inferred from the asymptotic period spacing $\left(M_{*}=0.597 M_{\odot}\right)$ (see Table 1). On the other hand, we note that $M_{*}$ for the best-fit model is in excellent agreement with the spectroscopic derivation of Miller Bertolami \& Althaus (2006) (0.542 $M_{\odot}$ versus $\left.0.53 M_{\odot}\right)$, but is substantially lower than the spectroscopic value

\footnotetext{
2 We elect the value of the spectroscopic mass of PG 2131+066 inferred by Miller Bertolami \& Althaus (2006) for this comparison because they use the same post-born again PG 1159 evolutionary models we employ here in the determination of the asteroseismological mass, and because the spectroscopic masses quoted by Werner \& Herwig (2006) are based on old post-AGB tracks.
}

Table 5. Same as Table 4, but for PG $1707+427$.

\begin{tabular}{lccc}
\hline \hline Quantity & Spectroscopy & Seismology & This work \\
\hline$T_{\text {eff }}[\mathrm{kK}]$ & $85 \pm 4.5$ & - & $89.5_{-1.8}^{+1.7}$ \\
$M_{*}\left[M_{\odot}\right]$ & $0.59 \pm 0.1$ & $0.57 \pm 0.02$ & $0.542_{-0.012}^{+0.014}$ \\
& $(0.53 \pm 0.1)$ & & \\
$\log g\left[\mathrm{~cm} / \mathrm{s}^{2}\right]$ & $7.5 \pm 0.3$ & - & $7.53_{-0.08}^{+0.09}$ \\
$\log \left(L_{*} / L_{\odot}\right)$ & - & 1.36 & $1.40 \pm 0.04$ \\
$\log \left(R_{*} / R_{\odot}\right)$ & - & - & $-1.68 \pm 0.04$ \\
$M_{\text {env }}\left[M_{\odot}\right]$ & - & - & 0.025 \\
$M_{\mathrm{V}}[\mathrm{mag}]$ & - & - & $7.25 \pm 0.6$ \\
$M_{\mathrm{bol}}[\mathrm{mag}]$ & - & - & $1.25 \pm 0.1$ \\
$A_{\mathrm{V}}[\mathrm{mag}]$ & - & - & 0.12 \\
$d[\mathrm{pc}]$ & - & - & $730_{-170}^{+230}$ \\
$\pi[\mathrm{mas}]$ & - & - & $1.4 \pm 0.4$ \\
\hline
\end{tabular}

quoted by Werner \& Herwig (2006) $\left(0.59 M_{\odot}\right)$. Until now, the asteroseismological mass of PG $1707+427$ has been more than $7 \%$ larger $\left(\Delta M_{*} \approx 0.04 M_{\odot}\right)$ than the spectroscopic mass if we adopt for the seismological mass the value found by Kawaler et al. (2004) and the derivation of Miller Bertolami \& Althaus (2006) for the spectroscopic mass. In light of our best-fit model, this discrepancy is strongly reduced to about $2 \%\left(\Delta M_{*} \approx 0.012 M_{\odot}\right)$. Finally, our best-fit model for PG $1707+427$ is slightly more luminous than what is suggested by Kawaler et al. (2004).

\subsection{The asteroseismological distance and parallax of $P G 2131+066$ and $P G 1707+427$}

As in our previous works - see, e.g., Córsico et al. (2007a) we employ the luminosity of our best-fit models to infer the seismic distance to PG $2131+066$ and PG $1707+427$. Following Kawaler et al. (1995), we adopt $\mathrm{BC}=-6.0 \pm 0.5$ for both stars (Werner et al. 1991). We account for the interstellar absorption, $A_{\mathrm{V}}$, using the interstellar extinction model of Chen et al. (1998). With all these ingredients the seismic distance, $d$, can be easily computed using the apparent magnitudes, which are $m_{\mathrm{v}}=16.6$ for PG $2131+066$ and $m_{\mathrm{v}}=16.7$ for PG 1707+427 (Bond et al. 1984). We obtain a distance $d \sim 830 \mathrm{pc}$ and an interstellar extinction $A_{\mathrm{V}} \sim 0.18$ for PG $2131+066$ and $d \sim 730 \mathrm{pc}$ and $A_{\mathrm{V}} \sim 0.12$ for PG $1707+427$.

Our estimation of the distance to PG $2131+066$ is $\sim 15 \%$ larger than that derived by CA06 $(d \sim 716 \mathrm{pc})$. This is because our asteroseismological model is somewhat more luminous than that of CA06 $\left(\log \left(L_{*} / L_{\odot}\right)=1.57\right.$ versus $\left.\log \left(L_{*} / L_{\odot}\right)=1.37\right)$. On the other hand, our distance is 20-25\% larger than that obtained by Reed et al. (2000) ( $d \sim 668$ pc) on the basis of their own asteroseismological analysis. This difference can be understood on the basis that Reed et al. (2000) uses a luminosity of $\log \left(L_{*} / L_{\odot}\right) \sim 1.4$, somewhat lower than that of our best-fit model for PG $2131+066$, of $\log \left(L_{*} / L_{\odot}\right) \sim 1.6$. On the other hand, our asteroseismological distance for PG 2131+066 is about 1.2 times longer than that quoted by Reed et al. (2000) of $\sim 680 \mathrm{pc}$ obtained on the basis of spectrum fitting, although both estimations are compatible at the $1 \sigma$ level.

For PG $1707+427$, our asteroseismological distance is in agreement with that quoted by CA06, of $\sim 697 \mathrm{pc}$. Werner et al. (1991) obtain a distance to PG $1707+427$ of $\sim 1300$ pc, substantially larger than our estimation, but still within the quoted error bars. The different value of Werner et al. (1991) is due to that they use a luminosity of $\log L_{*} / L_{\odot}=2.15$, substantially higher than the luminosity of our best fit model $\left(\log L_{*} / L_{\odot}=1.4\right)$. 


\section{Comparison with the results of CA06}

Following the recommendations of an anonymous referee, we include in this section a detailed comparison between the PG 1159 models and the asteroseismological results of the present paper and those of the previous study by CA06. These authors performed an asteroseismological analysis of four GW Vir stars (PG 0122+200, PG 1159-035, PG 2131+066, and PG 1707+427) on the basis of a set of twelve PG 1159 evolutionary sequences with different stellar masses $\left(M_{*}=0.53,0.54,0.55, \ldots, 0.62,0.63,0.64 M_{\odot}\right)$ artificially derived from the full evolutionary sequence of $0.5895 M_{\odot}$ computed by Althaus et al. (2005). That sequence is one of the sequences we use in the present paper. Specifically, the sequences of CA06 were constructed using LPCODE by appropriately scaling the stellar mass of the $0.589 M_{\odot}$ sequence before the models reach the low-luminosity, high-gravity stage of the GW Vir domain. Although this procedure leads to a series of unphysical stellar models for which the helium-burning luminosity is not consistent with the thermo-mechanical structure, the transitory stage vanishes shortly before the star reaches the evolutionary "knee" in the HR diagram (see Fig. 2 of CA06). As a consequence, those PG 1159 models were not suitable for the highluminosity, low-gravity regime corresponding, for instance, to RX J2117.1+3412, NGC 1501, K 1-16, HE 1429-1209, etc.

Because the sequences of CA06 with different stellar masses were created starting from a single sequence with $M_{*}=$ $0.589 M_{\odot}$, the central abundances of $\mathrm{C}$ and $\mathrm{O}$ and the spatial extension of the $\mathrm{C}-\mathrm{O}$ core are not completely consistent with the value of the stellar mass, except in the case of the models of the $0.589 M_{\odot}$ sequence itself. For instance, a model of CA06 with $M_{*}=0.64 M_{\odot}$ has a $\mathrm{C}-\mathrm{O}$ core that is somewhat smaller and the central abundance of $\mathrm{O}$ is substantially higher than what would be expected if the complete evolution of the progenitor star were performed, as it is the case for the models employed in the present paper. Figure 2 of Miller Bertolami \& Althaus (2006) clearly illustrate that, when the complete evolution of the PG 1159 progenitor stars is taken into account, different stellar masses are associated with different central abundances of $\mathrm{C}$ and $\mathrm{O}$, and different sizes of the $\mathrm{C}-\mathrm{O}$ core. Specifically, the more massive the models, the lower (higher) the central abundance of $\mathrm{O}(\mathrm{C})$ and the larger the $\mathrm{C}-\mathrm{O}$ core. In summary, for a given value of $M_{*}$, and for stages after the evolutionary knee, the only structural/physical difference between the PG 1159 models employed in the present work and those of CA06 is related to the size of the $\mathrm{C}-\mathrm{O}$ core and the central abundances of $\mathrm{O}$ and $\mathrm{C}$.

In Fig. 4 we compare some evolutionary tracks $\left(M_{*}=0.530\right.$, $0.542,0.589,0.609 M_{\odot}$ ) of the PG 1159 sequences employed in the present work with the corresponding evolutionary tracks of CA06 $\left(M_{*}=0.53,0.54,0.59,0.61 M_{\odot}\right)$. A careful inspection of this figure reveals that both sets of tracks generally differ, but when the models reach the beginning of their white dwarf stage $\left(\log T_{\text {eff }} \lesssim 5\right)$, they turn be in very close agreement. This agreement is reached earlier in the case of sequences with stellar masses close to $M_{*}=0.589 M_{\odot}$, the value of the sequence from which the remainder sequences of CA06 were generated. Interestingly enough, the regime in which the tracks of CA06 are in agreement with those employed in the present paper embraces the location of PG $2131+066$ and PG 1707+427, which have $\log T_{\text {eff }}=4.97$ and $\log T_{\text {eff }}=4.93$, respectively. Because of this, it is expected that the global pulsation properties (i.e., asymptotic period spacing, average of the computed period spacing) of both sets of models in that regime should nearly agree, and consequently the asteroseismological inferences on PG 2131+066

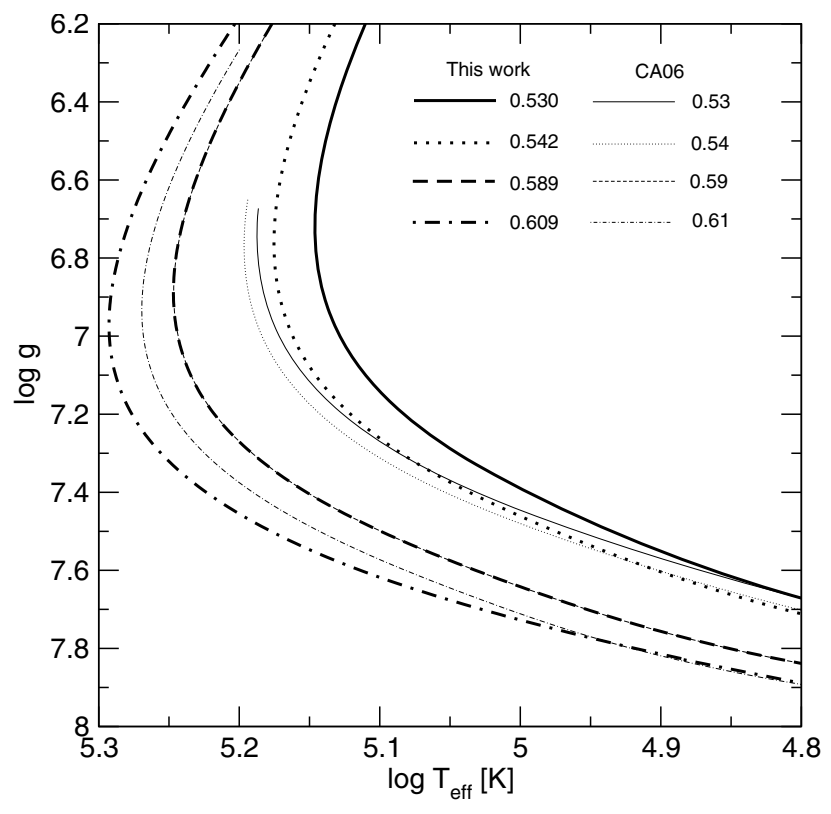

Fig. 4. Comparison between some PG 1159 evolutionary tracks employed in the present work (thick lines) and those of CA06 (thin lines). The values are in solar masses. Note that the track corresponding to the sequence of $\approx 0.59 M_{\odot}$ is the same for the two sets of computations.

Table 6. Comparison between the stellar mass values (in $M_{\odot}$ ) obtained in CA06 and in the present work.

\begin{tabular}{lccc}
\hline \hline PG 2131+066 & $\Delta \Pi_{\ell}^{\mathrm{a}}$ & $\overline{\Delta \Pi_{\ell}}$ & Period fit \\
\hline CA06 & 0.615 & 0.575 & 0.60 \\
This work & 0.627 & 0.578 & 0.589 \\
$\Delta M_{*} / M_{*}$ & $2 \%$ & $0.3 \%$ & $2 \%$ \\
\hline PG 1707+427 & $\Delta \Pi_{\ell}^{\mathrm{a}}$ & $\overline{\Delta \Pi_{\ell}}$ & Period fit \\
\hline CA06 & 0.595 & 0.565 & 0.55 \\
This work & 0.597 & 0.566 & 0.542 \\
$\Delta M_{*} / M_{*}$ & $0.3 \%$ & $0.1 \%$ & $1.5 \%$ \\
\hline
\end{tabular}

and PG $1707+427$ based on these two different sets of models should not be substantially distinct.

In Table 6 we present a comparison between the stellar masses inferred in CA06 (their Table 1) and in the present study. Note that, not surprisingly, the stellar masses derived in CA06 are in excellent agreement with the values obtained in the present work, irrespective of the particular method employed, being the differences in all of the cases below $2 \%$. This is a clear indication that the asteroseismological results of this paper and that of CA06 for PG $2131+066$ and PG $1707+427$ are not seriously affected by the differences between both sets of models. This adds credibility and robustness to the asteroseismological results of the present study. This conclusion should change for the case of PG 1159 pulsators with stellar masses too departed from the value $\sim 0.589 M_{\odot}$ and/or located at earlier evolutionary stages.

\section{Summary and conclusions}

In this paper we presented an asteroseismological study of the high-gravity, low-luminosity pulsating PG 1159 stars PG 2131+066 and PG 1707+427 and of the high-luminosity PNNV [WCE] star NGC 1501. This is the fourth article of a short series of studies aimed at exploring the internal structure and evolutionary status of pulsating PG 1159 stars which have been intensively observed through multi-site campaigns. Our 
analysis is based on the full PG 1159 evolutionary models of Althaus et al. (2005), Miller Bertolami \& Althaus (2006) and Córsico et al. (2006). These models represent a solid basis to analyze the evolutionary and pulsational properties of pre-white dwarf stars like PG 2131+066, PG 1707+427, and NGC 1501.

We first used the observed period-spacing data to obtain estimations of the stellar mass of PG 2131+066, PG 1707+427, and NGC 1501. The results are summarized in Table 1, where we provide a summary of results from the present and the previous works by us, and also from other pulsation and spectroscopic studies. We obtained three mass values for each star: the first one by comparing the observed period spacing with the asymptotic period spacing of our models (an inexpensive and widely used approach that does not involve pulsational calculations); the second one by comparing the observed period spacings with the average of the computed period spacing (an approach that requires of detailed period computations); and the third one on the basis of the approximate formula of Kawaler \& Bradley (1994), which is based on the behavior of the asymptotic period spacing of a large grid of quasi-evolutionary PG 1159 models. The first and the third approaches are almost equivalent, and lead to similar, somewhat overestimated values of the stellar mass for PG 2131+066 and PG 1707+427. The second approach, clearly more realistic, conducts to smaller values of $M_{*}$ (and closer to the spectroscopic inferences) in the case of PG 2131+066 and PG $1707+427$, and virtually the same $M_{*}$ value than that obtained from the first approach in the case of NGC 1501.

In the second part of our work, we sought for the models that best reproduce the individual observed periods of each star. The period fits were made on a grid of PG 1159 models with a quite fine resolution in effective temperature $\left(\Delta T_{\text {eff }} \sim\right.$ $10-30 \mathrm{~K})$ although admittedly coarse in stellar mass $\left(\Delta M_{*} \sim\right.$ 0.01-0.08 $M_{\odot}$ ). We found asteroseismological models only for PG 2131+066 and PG 1707+427. For NGC 1501 we were unable to find a clear and unambiguous seismological solution due to the existence of numerous and equivalent minima characterizing the quality function employed in the period-fit procedure. The pulsational properties of the "best-fit" models for PG 2131+066 and PG 1707+427 are summarized in Tables 2 and 3 , respectively. In particular, we predict the values of the rates of period change to be positive and in the range $(2-5) \times$ $10^{-12} \mathrm{~s} / \mathrm{s}$. Unfortunately, we have been unable to check the reality of this prediction because the lack of any measurement

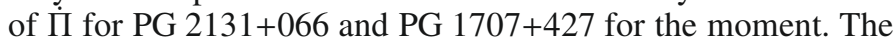
structural characteristics of these best-fit models are shown in Tables 4 and 5. In particular, the seismological masses are closer to the spectroscopic ones in light of our best-fit models, as we found in our previous works. In these tables we also show the seismological distances and parallaxes of PG 2131+066 and PG $1707+427$. We found a reasonable agreement between our results and those of Kawaler et al. (1995), Reed et al. (2000), Kawaler et al. (2004), and CA06. We stress that almost all differences between our results (Sects. 4.2 and 4.3) and those of earlier works are within the quoted errors.

In summary, in this work we have been able to estimate the stellar mass of PG 2131+066, PG 1707+427, and NGC 1501 on the basis of the period-spacing information alone. We have also been successful in finding asteroseismological models for PG 2131+066 and PG 1707+427 from period-to-period comparisons. In particular, the $T_{\text {eff }}$ and $\log g$ of the best-fit models are in very good agreement with the spectroscopic measurements. Unfortunately, we fail to found an asteroseismological model for NGC 1501. In principle, this shortcoming of our study could be indicating some inadequacy inherent to the stellar modeling. Another possible alternative could be the fact that the period spectrum of NGC 1501 includes periodicities associated with $g$-modes with radial orders very spaced from each other, in such a way that our $\chi^{2}$ procedure of period-fit is inefficient to isolate a clear and unambiguous asteroseismological solution. On the other hand, it would be kept in mind that while both PG 2131+066 and PG 1707+427 are classified as PG 1159 stars, NGC 1501 is a [WCE] star. Although both classes are suspected to form an evolutionary sequence, this possibility is still under debate (Crowther 2008; Todt et al. 2008) and it could not be the case. Therefore, the failure of our models to fit the period spectrum of NGC 1501 might be indicative that this star has a very different evolutionary history than PG 2131+066 and PG $1707+427$.

We have also included a comparison between the models and results of the present work and those of the study by CA06. The models employed in the present work are the result of the complete evolution of the progenitor stars, and as a result, they are characterized by central chemical abundances and a size of the $\mathrm{C}-\mathrm{O}$ core which are consistent with the value of the stellar mass. This is not the case for the models employed by CA06, which were artificially derived from the full evolutionary sequence of $0.589 M_{\odot}$ computed by Althaus et al. (2005). In spite of these differences, our asteroseismological results are in excellent agreement with those of CA06. This adds credibility and robustness to the results of the present study.

As the main conclusions of the present work, we can mention:

- The full evolutionary models of PG 1159 stars employed in the present work lead to asteroseismological results for PG 2131+066 and PG 1707+427 that do not differ substantially from those predicted by CA06 on the basis of evolutionary sequences generated artificially. We note, however, that this agreement between both sets of computations is valid for PG 1159 stars located at the low-luminosity, highgravity regime after the stars have passed the evolutionary knee in the HR diagram. It should be kept in mind that this conclusion should change for the case of stars located at earlier stages of evolution.

- At present, the PG 1159 evolutionary models used in this work - and in the previous studies of this series - remain the only suitable for asteroseismological inferences on stars that are located at the high-luminosity, low-gravity regime before the evolutionary knee, such as RX J2117.1+3412 and NGC 1501.

- The detailed fitting of the individual periods (Sect. 4) gives somewhat different masses than analysis based on asymptotic period spacing (methods 1 and 3 of Sect. 3), but in very good agreement with the values of $M_{*}$ derived from the average of the computed period spacings (method 2 of Sect. 3 ). Thus, method 2 is a very appropriate way to estimating stellar masses, and detailed period fits do not significantly improve the mass determinations. We note, however, that the period-fit approach yields an asteroseismological model from which one can infer, in addition to $M_{*}$, the luminosity, radius, gravity, and distance of the target star. In addition, the period-fit approach does not require - in principle - external constraints such as the spectroscopic values of $T_{\text {eff }}$ and $g$, i.e., the method works "by letting the pulsation modes speak for themselves" (see Metcalfe 2005, for an interesting discussion about this).

- The nonadiabatic stability analysis does not at the moment predict instability for all of the fitted modes. This means 
that, in the frame of the linear nonadiabatic pulsation theory, some pulsation modes detected in PG $2131+066$ and PG $1707+427$ should not be excited. It is not clear at this stage the origin of this discrepancy. Maybe it could be attributed to the extreme sensitivity of the stability analysis of PG 1159 stars to the exact amounts of the main atmospheric constituents (see Quirion et al. 2004, for details).

- The main conclusion of this series of papers - the present work and Córsico et al. (2007a,b, 2008) - is that for most well-observed pulsating PG 1159 stars (RX J2117.1+3412, PG 0122+200, PG 1159-035, PG 2131+066, and PG 1707+427) it is possible to found a stellar model (the asteroseismological model) with $M_{*}$ and $T_{\text {eff }}$ near the spectroscopic measurements to a high internal accuracy. The next step is of course an assessment of the question if the asteroseismological models can provide more accurate masses for these objects. The scatter in the masses derived from the different asteroseismological methods (see Table 1) suggests that it may not be the case. In fact, when all asteroseismological methods are considered, the uncertainty in the determination of the mass amounts to $\sim 0.05 M_{\odot}$, comparable to the spectroscopic one $\left(\sim 0.05-0.1 M_{\odot}\right.$, Werner et al. 2008). However, it is worth noting that, when results based on asymptotic period spacing (an approach that is not correct for the high-gravity regime of PG 1159 stars; see Althaus et al. 2008a) are not taken into account, the scattering in the derived masses is of only $\sim 0.02 M_{\odot}$.

We close the paper by noting that the PG 1159 evolutionary models employed in our series of asteroseismological studies are characterized by thick He-rich outer envelopes, as they are predicted by the standard theory for the formation of PG 1159 stars. However, Althaus et al. (2008b) have recently demonstrated that the assumption of thinner He-rich envelopes solves the longstanding discrepancy between the measured rates of period change in the prototypical star PG 1159-035 and the predictions of theoretical models. In view of this important result, we are planning future asteroseismological studies for all the pulsating PG 1159 stars analyzed in our series of articles, but with non-canonical PG 1159 models characterized by thinner He-rich envelopes.

Acknowledgements. Part of this work was supported by AGENCIA through the Programa de Modernización Tecnológica BID 1728/OC-AR, by the PIP 6521 grant from CONICET, by the MEC grant AYA2008-04211-C02-01, by the European Union FEDER funds, and by the AGAUR. This research has made use of NASA's Astrophysics Data System.

\section{References}

Althaus, L. G., Serenelli, A. M., Panei, J. A., et al. 2005, A\&A, 435, 631 Althaus, L. G., Córsico, A. H., Kepler, S. O., \& Miller Bertolami, M. M. 2008a, A\&A, 478, 175

Althaus, L. G., Córsico, A. H., Miller Bertolami, M. M., García-Berro, E., \& Kepler, S. O. 2008b, ApJ, 677, L35

Bond, H. E., \& Ciardullo, R. 1993, in White Dwarfs: Advances in Observations and Theory, ed. M. Barstow (Dordrecht: Kluwer), 491

Bond, H. E., Grauer, A. D., Green, R. F., \& Liebert, J. W. 1984, ApJ, 279, 751

Bond, H. E., Kawaler, S. D., Ciardullo, R., et al. 1996, AJ, 112, 2699

Chen, B., Vergely, J. L., Valette, B., \& Carraro, G. 1998, A\&A, 336, 137

Córsico, A. H., \& Althaus, L. G. 2006, A\&A, 454, 863

Córsico, A. H., Althaus, L. G., \& Miller Bertolami, M. M. 2006, A\&A, 458, 259

Córsico, A. H., Althaus, L. G., Miller Bertolami, M. M., \& Werner, K. 2007a, A\&A, 461, 1095

Córsico, A. H., Miller Bertolami, M. M., Althaus, L. G., Vauclair, G., \& Werner, K. 2007b, A\&A, 475, 619

Córsico, A. H., Althaus, L. G., Kepler, S. O., Costa, J. E. S., \& Miller Bertolami, M. M. 2008, A\&A, 478, 869

Costa, J. E. S., \& Kepler, S. O. 2008, A\&A, 489, 1225

Costa, J. E. S., Kepler, S. O., \& Winget, D. E. 1999, ApJ, 522, 973

Costa, J. E. S., Kepler, S. O., Winget, D. E., et al. 2008, A\&A, 477, 627

Crowther, P. A. 2008, ASP Conf. Ser., 391, 83

Dreizler, S., \& Heber, U. 1998, A\&A, 334, 618

Fontaine, G., \& Brassard, P. 2008, PASP, 120, 1043

Fu, J.-N., Vauclair, G., Solheim, J.-E., et al. 2007, A\&A, 467, 237

Herwig, F., Blöcker, T., Langer, N., \& Driebe, T. 1999, A\&A, 349, L5

Iben, I. Jr., Kaler, J. B., Truran, J. W., \& Renzini, A. 1983, ApJ, 264, 605

Kawaler, S. D., \& Bradley, P. A. 1994, ApJ, 427, 415

Kawaler, S. D., O'Brien, M. S., Clemens, J. C., et al. 1995, ApJ, 450, 350

Kawaler, S. D., Potter, E. M., Vucković, M., et al. 2004, A\&A, 428, 969

Koesterke, L., \& Hamann, W. R. 1997, A\&A, 320, 91

Lawlor, T. M., \& MacDonald, J. 2003, ApJ, 583, 913

Metcalfe, T. S. 2005, JApA, 26, 273

Miller Bertolami, M. M., \& Althaus, L. G. 2006, A\&A, 454, 845

Miller Bertolami, M. M., \& Althaus, L. G. 2007a, A\&A, 470, 675

Miller Bertolami, M. M., \& Althaus, L. G. 2007b, MNRAS, 380, 763

Nather, R. E., Winget, D. E., Clemens, J. C., Hansen, C. J., \& Hine, B. P. 1990, ApJ, 361, 309

Quirion, P.-O., Fontaine, G., \& Brassard, P. 2004, ApJ, 610, 436

Reed, M. D., Kawaler, S. D., \& O’Brien, M. S. 2000, ApJ, 545, 429

Tassoul, M., Fontaine, G., \& Winget, D. E. 1990, ApJS, 72, 335

Todt, H., Peña, M., Hamann, W.-R., \& Gräfener, G. 2008, ASP Conf. Ser., 391, 95

Townsley, D. M., Arras, P., \& Bildstein, L. 2004, ApJ, 608, L105

Vauclair, G., Moskalik, P., Pfeiffer, B., et al. 2002, A\&A, 381, 122

Werner, K., \& Herwig, F. 2006, PASP, 118, 183

Werner, K., Heber, U., \& Hunger, K. 1991, A\&A, 244, 437

Werner, K., Rauch, T., Reiff, E., \& Kruk, J. W. 2008, Hydrogen Defficient Stars, ASP Conf. Ser., 391, 109

Winget, D. E., \& Kepler, S. O. 2008, ARA\&A, 46, 157 